\title{
Integral transforms for three-dimensional pumping in confined, leaky, and unconfined aquifers
}

\author{
Elizeu Melo da Silva ${ }^{1,2}$, João N. N. Quaresma ${ }^{1,3^{*}}$, Emanuel N. Macêdo ${ }^{1,3}$, Renato M. Cotta ${ }^{4,5}$ \\ ${ }^{1}$ Graduate Program in Natural Resources Engineering in the Amazon, PRODERNA/ITEC/UFPA, Universidade Federal do Pará, \\ 66075-110, Belém, PA, Brazil. \\ ${ }^{2}$ School of Mining Engineering, UNIFESSPA, Universidade Federal do Sul e Sudeste do Pará, CEP 68505-080, Marabá, PA, Brazil. \\ ${ }^{3}$ School of Chemical Engineering, FEQ/ITEC/UFPA, Universidade Federal do Pará, 66075-110, Belém, PA, Brazil. \\ ${ }^{4}$ General Directorate of Nuclear and Technological Development, DGDNTM, Marinha do Brasil, Rio de Janeiro, RJ, Brazil. \\ ${ }^{5}$ Department of Mechanical Engineering, POLI \& COPPE, CT, Universidade Federal do Rio de Janeiro, Rio de Janeiro, RJ, Brazil. \\ * Corresponding author. E-mail: quaresma@ufpa.br
}

\begin{abstract}
Analytical or hybrid numerical-analytical solutions based on the Generalized Integral Transform Technique (GITT) are obtained for the transient three-dimensional pumping problem of aquifers with a fully penetrating vertical well between two parallel streams. The problem formulation for confined and leaky aquifers allows for achieving exact analytical solutions through integral transforms, while the unconfined aquifer case introduces a fourth kind boundary condition which leads to a coupled transformed head ordinary differential system, that can be solved either analytically or numerically. A convergence analysis is performed to illustrate the consistency of the numerical results achieved for the head distribution, as well as for the related pumping rates. Results are obtained for selected cases and comparisons with literature results are performed. A solution verification confirms the agreement of the integral transform solutions with available simulations and provides additional confidence for the analysis of a few physical parameters that influence the hydrological behavior.
\end{abstract}

Keywords: Integral transforms; Hybrid methods; Aquifer pumping problem; Head distribution; Stream depletion rate.

\section{INTRODUCTION}

The water collected in wells near streams can reduce the flow in a stream through a process known as streamflow depletion. This problem has become particularly important in recent years, especially around densely populated regions, due to the significant increase in water resources demands for supplying large cities, large agricultural areas, and other uses, such as processing industries or mining activities (Knowling and Werner, 2016; Loizeau et al., 2017; Pastore et al., 2017). For this reason, regulatory agencies seek to establish the maximum groundwater pumping for wells located near streams. The pumping near streams causes depletion of the river to the aquifer, characterized by a stream depletion rate (SDR). Thus, more realistic solutions are required, leading to a better understanding of the SDR transient behavior under the pumping effect. According to Huang et al. (2014), the SDR only begins after the drawdown cone reaches the streambed once pumping has started. In the steady state, the water pumped out of the aquifer is solely from the infiltration of rivers through their semi-permeable beds.

The first studies about SDR were developed by Theis (1941). Glover and Balmer (1954) found a solution to the same problem, written in terms of the complementary error function, and the Theis solution was then renamed the Glover-Balmer solution. Hantush (1965) attempted to make the Theis problem slightly more realistic by aligning the edge flow with a semipermeable aquitard of semi-infinite length. Hunt (1999) obtained a solution to the two-dimensional problem of a partially penetrating stream with infinite length in a homogeneous Theis' aquifer, and later on, Hunt (2001) compared his solution to experimental results. Butler et al. (2001) proposed a solution to the depletion aquifer with semi-infinite geometry, caused by a full penetration vertical well adjacent to a limited lateral extension stream, i.e., the stream and aquifer both have finite width. The contributions reviewed above divide the domain into two different regions that contain a stream between them, while the governing equation for each region was determined by coupling the head and flow continuity at the interfaces between the intermediate zone and the lateral zones.

Other models of aquifers admit horizontal wells and have been developed for draining, which have been increasingly common, such as in the works of Hantush and Papadopoulos (1962), Zhan and Zlotnik (2002), Chen et al. (2003), Zhan and Park (2003), Sun and Zhan (2006), Huang et al. (2011), Huang et al. (2012a), and Huang et al. (2016).

Most of the analytical work has been developed in the semiinfinite domain, and the solutions are commonly given in the time or Laplace-domains. Analytical solutions for multilayer configurations or more general heterogeneous aquifer problems remain scarce in the literature. However, some researchers include the assumption of soil heterogeneity as layers of different physical properties, such as in Butler et al. (2007), Malama et al. (2007), Hunt (2009), and Ward and Lough (2011).

Strack (1999, 2003), Bakker and Strack (2003), Bakker (2004), and Bakker et al. (2005) used the analytic elements method for the mathematical modeling of groundwater flow. The technique resembles the classical boundary element method but differs through the analytical nature of the chosen solutions. Sun and Zhan (2007) modelled the two-dimensional problem in a fully penetrating vertical well located between two parallel streams, simulating the streambed effect as a lowpermeability contour, analyzing how the streambed affects the stream depletion rate, and obtaining the solution by Laplace transform. Similarly, Intaraprasong and Zhan (2007) applied 
the Fourier transform to obtain the solution of the twodimensional steady-state problem. Huang et al. (2012b) solved the problem of a two-dimensional leaky aquifer, while Huang et al. (2014) presented a model of the three-dimensional problem to describe the groundwater flow induced by a fully penetrating vertical well on aquifers between two parallel streams. A general formulation was considered to represent the upper contour boundary condition directly applicable to the confined, leaky, or unconfined aquifer cases. The solution proposed by Huang et al. (2014) is given by Laplace transform, and inversions for the time domain are obtained analytically for the confined case. However, for the leaky and unconfined cases, the Padé approximant is used to obtain the solutions, which are then compared to a numerical solution obtained by Crump's method.

There is no questioning about the advantages of obtaining analytical solutions for partial differential equations, once the specific problem formulation allows for, due to the inherent full precision control and the resulting minimal computational cost. The availability of an analytical expression directly offers information at any specific location of the domain and mathematical operations such as differentiation and integration are readily implemented. Besides, analytical solutions permit the inspection of asymptotic trends and parametric dependences with very little or no numerical calculation effort. Although numerical methods are, in principle, more flexible and straightforward, numerical error propagation and instabilities may eventually impair (or at least bring up costly mesh refinement) the universal use of the traditional computational approaches, such as in problems with asymptotic behaviors and/or abrupt variations. For this reason, hybrid numericalanalytical approaches find a niche of application, providing a bridge between classical analytical methods and modern numerical analysis. The most successful hybrid methods lead to computational-analytical algorithms that still offer error control within mild computational effort. The range of problems that can provide sets of benchmark independent results for verification of numerical codes is then naturally and widely extended. In addition, once enough flexibility is incorporated into the hybrid approach algorithm, it may serve itself as an alternative computational path for different classes of problems. Here, the formulation proposed by Huang et al. (2014) is revisited but a different solution path is considered through application of the Generalized Integral Transform Technique (GITT) (Cotta, 1990, 1993, 1994, 1998; Cotta and Mikhailov, 1997, 2006; Cotta et al., 2013, 2014, 2018a, 2018b; Santos et al., 2001). The GITT methodology consists of reducing partial differential equations (PDE) to an infinite system of coupled ordinary differential equations (ODE) for the transformed potentials through integral transformation, which is then either solved analytically for certain linear problems, thus yielding an exact solution, or numerically for both coupled linear or nonlinear problems, then providing a hybrid numericalanalytical solution. Besides the general reference books and review papers above mentioned, a few previous works are here pointed out that employed the GITT on streams and groundwater flow and contaminant dispersion problems, such as Almeida and Cotta (1995, 1996a), Liu et al. (2000), Cotta et al. (2003), Barros et al. (2006), Barros and Cotta (2007), Naveira-Cotta et al. (2013), Cotta et al. (2018c). Also, the nature of the source terms here to be analyzed and the threedimensional geometry under consideration require some sort of convergence acceleration techniques for the computational implementation of the proposed eigenfunction expansions. Therefore, filtering solutions are employed (Almeida and Cotta, 1996b; Cotta, 1993; Cotta and Mikhailov, 1997; Cotta et al., 2013, 2014), together with reordering strategies for multiple expansions (Correa et al., 1997; Cotta et al., 2013, 2014; Mikhailov and Cotta, 1996), and the resulting convergence behavior is critically analyzed.

\section{MATHEMATICAL FORMULATION}

The mathematical model that describes the pumping problem using a fully penetrating vertical well is the same as proposed by Huang et al. (2014). The governing equation for a three-dimensional transient hydraulic head distribution, $h(x, y, z, t)$, in a homogeneous and anisotropic aquifer, induced by a fully penetrating vertical well positioned at point $\left(x_{0}, y_{0}\right)$, is expressed in dimensionless form as:

$$
\begin{aligned}
& \frac{\partial h}{\partial t}=\frac{\partial^{2} h}{\partial x^{2}}+\frac{\partial^{2} h}{\partial y^{2}}+k_{v} \frac{\partial^{2} h}{\partial z^{2}}-\delta(x-1) \delta\left(y-y_{0}\right) \\
& 0<x<w_{x}, 0<y<w_{y}, 0<z<1, \text { and } t>0 \\
& h(x, y, z, 0)=0 \\
& \frac{\partial h(0, y, z, t)}{\partial x}-k_{1} h(0, y, z, t)=0 ; \\
& \frac{\partial h\left(w_{x}, y, z, t\right)}{\partial x}+k_{2} h\left(w_{x}, y, z, t\right)=0 \\
& \frac{\partial h(x, 0, z, t)}{\partial y}=0 ; \quad \frac{\partial h\left(x, w_{y}, z, t\right)}{\partial y}=0 \\
& \frac{\partial h(x, y, 0, t)}{\partial z}=0 ; \\
& \frac{\partial h(x, y, 1, t)}{\partial z}+\frac{\sigma}{k_{v}} \frac{\partial h(x, y, 1, t)}{\partial t}+k^{\prime} h(x, y, 1, t)=0
\end{aligned}
$$

where the following dimensionless groups were adopted:

$$
\begin{aligned}
& h=\frac{K_{h} H}{Q} h^{*} ; \quad x=\frac{x^{*}}{x_{0}^{*}} ; \quad y=\frac{y^{*}}{x_{0}^{*}} ; \quad z=\frac{z^{*}}{H} ; \\
& y_{0}=\frac{y_{0}^{*}}{x_{0}^{*}} ; \quad t=\frac{K_{h}}{S_{S} x_{0}^{* 2}} t^{*} ; \quad \sigma=\frac{S_{y}}{S_{s} H} ; \\
& k_{v}=\frac{K_{v} x_{0}^{* 2}}{H^{2} K_{h}} ; \quad k^{\prime}=\frac{K^{\prime} H}{B^{\prime} K_{v}} ; \quad k_{1}=\frac{K_{1} x_{0}^{*}}{B_{1} K_{h}} ; \\
& k_{2}=\frac{K_{2} x_{0}^{*}}{B_{2} K_{h}} ; \quad w_{x}=\frac{W_{x}}{x_{0}^{*}} ; \quad w_{y}=\frac{W_{y}}{x_{0}^{*}}
\end{aligned}
$$

The boundary condition, Eq. (1h), offers three different aquifer models:

- Confined aquifer, if $\sigma=0$ and $k^{\prime}=0$;

- Leaky aquifer, if $\sigma=0$ and $k^{\prime}>0$;

- Unconfined aquifer, if $\sigma>0$ and $k^{\prime}=0$.

Equation (1) governs the three-dimensional transient head behavior in an aquifer with thickness $H$ and specific storage $S_{s}$ that contains a fully penetrating vertical well to a drainage rate $Q$ between two parallel streams of negligible width. The streams near the well are partially isolated by the streambeds, with the conductivities and thicknesses denoted by $\left(K_{1}, B_{1}\right)$ and $\left(K_{2}, B_{2}\right)$, respectively, as proposed by Sun and Zhan (2007) and 
Intaraprasong and Zhan (2007). The aquifer has a finite width in the $x$ and $y$ directions, denoted by $W_{x}$ and $W_{y}$, respectively. The proposed model also allows for the existence of an aquitard of thickness $B^{\prime}$ with conductivity $K^{\prime}$ between the aquifer and an overlying adjacent aquifer. The well is located at $\left(x_{0}{ }^{*}, y_{0}{ }^{*}\right)$, as shown schematically in Fig. 1(a), indicating the well horizontal position. The z-direction of the aquifer depth has a top boundary condition capable of defining the three types of aquifers, as mentioned above: confined, Fig. 1(b), leaking, Fig. 1(c), and unconfined, Fig. 1(d), depending on the conductivity of the aquitard $K^{\prime}$ between aquifers and their specific yield $S_{y}$. When the conductivity $K^{\prime}$ is exceptionally low, the aquitard becomes an aquiclude and the aquifer is then confined. The model describes an anisotropic medium. Therefore, the hydraulic conductivity on the aquifer is equal on the $x$ and $y$ directions, $K_{h}$, but different in the z-direction, $K_{v}$.

Equation (1h) generalizes the boundary condition at the top that models the three different types of aquifers: confined, leaky and unconfined for the problem here analyzed, where $S_{y}$ is the specific yield of unconfined aquifers, $K^{\prime}$ is the vertical hydraulic conductivity of the connected aquitard of thickness $B$. The general boundary condition $(1 \mathrm{~h})$ simulates a leaky aquifer if the specific yield $S_{y}=0$, containing an aquitard of width $B$ with conductivity $K^{\prime}>0$ between the aquifer and an overlying aquifer. It also models a confined aquifer if the aquitard conductivity is too low, i.e., $K^{\prime}=0$. When $K^{\prime}$ is low, the aquitard becomes an aquiclude or an impermeable layer, passing to a non-flow condition and if no have specific yield, $S_{y}=0$, models a confined aquifer. Finally, if $S_{y}>0$ and $K^{\prime}=0$, the equation models an unconfined aquifer, disregarding the existence of an aquitard among the adjacent aquifers, making a single aquifer.

\section{SOLUTION METHODOLOGY}

In this work, the Generalized Integral Transform Technique (GITT), Cotta (1990, 1993, 1994, 1998), Cotta and Mikhailov (1997, 2006), Santos et al. (2001), Cotta et al. (2013, 2014, $2018 \mathrm{a}, 2018 \mathrm{~b}$ ), is applied to the proposed model. The GITT automatically reduces to the classical integral transform method (Özisik, 1980; Mikhailov and Özisik, 1984) once the transformed problem becomes a decoupled linear ODE system. Otherwise, when an exact analytical solution cannot be recovered, the generalized approach still provides hybrid numericalanalytical solutions based on the numerical solution of a truncated transformed ODE system.

For convergence rates improvement, the first step in the GITT formalism is the proposition of a filtering solution to reduce the importance of source terms in the eigenfunction expansions convergence. It is well known that optimal convergence rates in eigenfunction expansions are achieved in homogeneous problems, such as in the solutions achieved through separation of variables, characterized by summation terms with an exponential decay with the squared eigenvalue as argument. For nonhomogeneous problems, this exponential behavior does not prevail, and a typical rational pattern in terms of the inverse of the squared eigenvalues can markedly slow down the overall expansion convergence. Thus, it pays back to filter the original problem with known analytical solutions of simpler formulations that account for the source terms in the equations and boundary conditions, so as to reduce the relative importance of these nonhomogeneous terms in the final expression for the expansion terms. For this purpose, a filter is here proposed that represents the steady state solution of the three-dimensional head distribution, in the form:
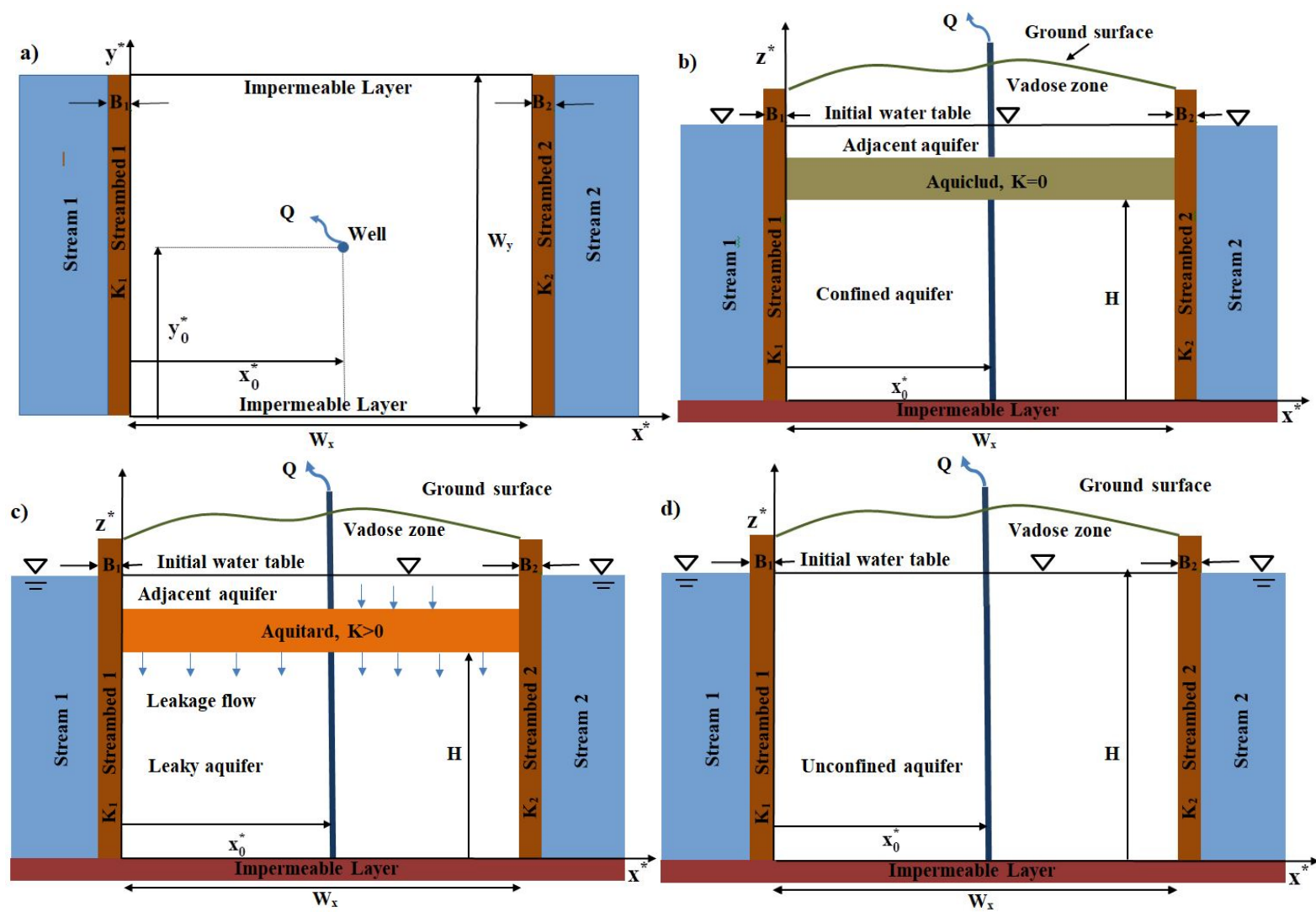

Fig. 1. Schematic representation of hydraulic head distribution in an aquifer between two rivers being drained by a fully penetrating vertical well: (a) well position; (b) confined aquifer; (c) leaky aquifer; (d) unconfined aquifer. 


$$
h(x, y, z, t)=h_{H}(x, y, z, t)+h_{f}(x, y, z)
$$

Writing the filter formulation and substituting Eq. (3) into Eqs. (1), the two distinct problems are obtained as follows:

- Proposed filter problem:

$$
\begin{aligned}
& \frac{\partial^{2} h_{f}}{\partial x^{2}}+\frac{\partial^{2} h_{f}}{\partial y^{2}}+k_{v} \frac{\partial^{2} h_{f}}{\partial z^{2}}=\delta(x-1) \delta\left(y-y_{0}\right) \\
& 0<x<w_{x}, 0<y<w_{y}, \text { and } 0<z<1 \\
& \frac{\partial h_{f}(0, y, z)}{\partial x}-k_{1} h_{f}(0, y, z)=0 ; \\
& \frac{\partial h_{f}\left(w_{x}, y, z\right)}{\partial x}+k_{2} h_{f}\left(w_{x}, y, z\right)=0 \\
& \frac{\partial h_{f}(x, 0, z)}{\partial y}=0 ; \quad \frac{\partial h_{f}\left(x, w_{y}, z\right)}{\partial y}=0 \\
& \frac{\partial h_{f}(x, y, 0)}{\partial z}=0 ; \quad \frac{\partial h_{f}(x, y, 1)}{\partial z}+k^{\prime} h_{f}(x, y, 1)=0
\end{aligned}
$$

- Filtered homogeneous problem:

$$
\begin{aligned}
& \frac{\partial h_{H}}{\partial t}=\frac{\partial^{2} h_{H}}{\partial x^{2}}+\frac{\partial^{2} h_{H}}{\partial y^{2}}+k_{v} \frac{\partial^{2} h_{H}}{\partial z^{2}} \\
& 0<x<w_{x}, 0<y<w_{y}, 0<z<1, \text { and } t>0, \\
& h_{H}(x, y, z, 0)=-h_{f}(x, y, z) \\
& \frac{\partial h_{H}(0, y, z, t)}{\partial x}-k_{1} h_{H}(0, y, z, t)=0 ; \\
& \frac{\partial h_{H}\left(w_{x}, y, z, t\right)}{\partial x}+k_{2} h_{H}\left(w_{x}, y, z, t\right)=0 \\
& \frac{\partial h_{H}(x, 0, z, t)}{\partial y}=0 ; \quad \frac{\partial h_{H}\left(x, w_{y}, z, t\right)}{\partial y}=0 \\
& \frac{\partial h_{H}(x, y, 0, t)}{\partial \mathrm{z}}=0 ; \\
& \frac{\partial h_{H}(x, y, 1, t)}{\partial z}+\frac{\sigma}{k_{v}} \frac{\partial h_{H}(x, y, 1, t)}{\partial t}+k^{\prime} h_{H}(x, y, 1, t)=0
\end{aligned}
$$

\section{Homogeneous problem solution}

The solution for the homogeneous problem is obtained through an extension of the GITT approach (Cotta, 1993). In principle, an exact integral transform solution could be derived from the general theory for fourth and fifth kind boundary conditions as first proposed in (Mikhailov and Özisik, 1985), once the corresponding three-dimensional eigenvalue problem is obtained by Separation of Variables from Eqs. (5) and then solved by the GITT itself. Here choosing a simpler threedimensional eigenvalue problem that is uncoupled in the three coordinates, which allows for the straightforward definition of the three-dimensional eigenfunction expansion, given by:

$$
\frac{\partial^{2} \psi_{i}}{\partial x^{2}}+\frac{\partial^{2} \psi_{i}}{\partial y^{2}}+k_{v} \frac{\partial^{2} \psi_{i}}{\partial z^{2}}+\mu_{i}^{2} \psi_{i}(x, y, z)=0
$$

$$
\begin{aligned}
& \frac{\partial \psi_{i}(0, y, z)}{\partial x}-k_{1} \psi_{i}(0, y, z)=0 \\
& \frac{\partial \psi_{i}\left(w_{x}, y, z\right)}{\partial x}+k_{2} \psi_{i}\left(w_{x}, y, z\right)=0 \\
& \frac{\partial \psi_{i}(x, 0, z)}{\partial y}=0 ; \quad \frac{\partial \psi_{i}\left(x, w_{y}, z\right)}{\partial y}=0 \\
& \frac{\partial \psi_{i}(x, y, 0)}{\partial z}=0 ; \quad \frac{\partial \psi_{i}(x, y, 1)}{\partial z}+\left(k^{\prime}-\frac{\sigma}{k_{v}} \gamma_{r}^{2}\right) \psi_{i}(x, y, 1)=0
\end{aligned}
$$

The problem described above is called the Sturm-Liouville problem and its solution is obtained through expansions of eigenvalues that equally satisfy the boundary conditions. Its solution is later obtained through the superposition of the linearly independent eigenfunctions in a series that converges when the number of used eigenvalues is sufficiently large. The threedimensional eigenfunction $\psi_{i}(x, y, z)=X_{m}(x) Y_{p}(y) Z_{r}(z)$ corresponds to the product of the one-dimensional eigenfunctions in directions $x, y$ and $z$, and the three indices combination provides the single index $\mathrm{i}(m, p, r \rightarrow i)$, and the corresponding eigenvalue of the three-dimensional eigenfunction becomes the sum of the squared eigenvalues corresponding to each one-dimensional eigenfunction, $\alpha_{m}^{2}+\beta_{p}^{2}+\gamma_{r}^{2}=\mu_{i}^{2}$. The separated one-dimensional eigenvalue problems and their corresponding solutions are shown in Table 1. It should be noticed that the eigenvalue problem separation was possible due to the choice of writing the boundary condition, Eq. (6g), only with the eigenvalue corresponding to the $\mathrm{z}$ coordinate auxiliary eigenvalue problem, instead of the global eigenvalue $\mu$.

To reduce computational costs, a reordering scheme of the eigenvalues was implemented according to Mikhailov and Cotta (1996) and Cotta and Mikhailov (1997), reducing the 3D combination of eigenvalues $\alpha_{m}^{2}+\beta_{p}^{2}+\gamma_{r}^{2}$ to the single squared eigenvalue $\mu_{i}^{2}$. The reordering scheme reduces the indices, selecting the smallest values obtained from the sums of the squared one-dimensional eigenvalues. This procedure is based on the principle that the convergence of such series depends mainly on the lower positive arguments that govern the exponential behavior of the time-dependent term (Cotta and Mikhailov, 1997), thus, the appropriate order of choosing these combinations is from lowest to highest values of the t-variable exponential arguments. The major contribution of this procedure is to reduce the multiple summations resulting from the transformation to a single summation dependent on a single index. An algorithm for reordering eigenvalues was developed by Mikhailov and Cotta (1996) for solving multi-dimensional diffusion problems. Cotta and Mikhailov (1997) also described this process applied to heat transfer problems using symbolic computation to obtain these sorted eigenvalues.

The presence of the squared eigenvalue in the boundary condition, Eq. (6g), makes problem (6) a non-classical eigenvalue problem, which requires the definition of a modified orthogonality condition, as proposed in Mikhailov and Özisik (1985). Then, problem (6) requires the definition of the following integral transform pair:

$$
\begin{aligned}
& \bar{h}_{i}(t)=\int_{0}^{w_{x}} \int_{0}^{w_{y}} \int_{0}^{1} \psi_{i}(x, y, z) h_{H}(x, y, z, t) \mathrm{d} z \mathrm{~d} y \mathrm{~d} x+ \\
& +\sigma \int_{0}^{w_{x}} \int_{0}^{w_{y}} \psi_{i}(x, y, 1) h_{H}(x, y, 1, t) \mathrm{d} y \mathrm{~d} x
\end{aligned}
$$




$$
h_{H}(x, y, z, t)=\sum_{i=1}^{\infty} \tilde{\Psi}_{i}(x, y, z) \bar{h}_{i}(t), \text { inverse }
$$

where

$$
N_{i}=N x_{m} \cdot N y_{p} \cdot N z_{r}, \quad \tilde{\psi}_{i}(x, y, z)=\frac{\psi_{i}(x, y, z)}{N_{i}}
$$

The integral transformation is accomplished through operation over Eq. (5a) and the initial condition (5b) employing both components of the transformation formula, Eq. (7a), through the transformation kernel, $\int_{0}^{w_{x}} \int_{0}^{w_{y}} \int_{0}^{1} \psi_{i}(x, y, z) \mathrm{d} z \mathrm{~d} y \mathrm{~d} x$, which is the operator used for equation transform as

$\int_{0}^{w_{x}} \int_{0}^{w_{y}} \int_{0}^{1} \psi_{i} \frac{\partial h_{H}}{\partial t} \mathrm{~d} z \mathrm{~d} y \mathrm{~d} x=\int_{0}^{w_{x}} \int_{0}^{w_{y}} \int_{0}^{1} \psi_{i} \frac{\partial^{2} h_{H}}{\partial x^{2}} \mathrm{~d} z \mathrm{~d} y \mathrm{~d} x+$ $+\int_{0}^{w_{x}} \int_{0}^{w_{y}} \int_{0}^{1} \psi_{i} \frac{\partial^{2} h_{H}}{\partial y^{2}} \mathrm{~d} z \mathrm{~d} y \mathrm{~d} x+k_{v} \int_{0}^{w_{x}} \int_{0}^{w_{y}} \int_{0}^{1} \psi_{i} \frac{\partial^{2} h_{H}}{\partial z^{2}} \mathrm{~d} z \mathrm{~d} y \mathrm{~d} x$

$\int_{0}^{w_{x}} \int_{0}^{w_{y}} \int_{0}^{1} \psi_{i} h_{H}(x, y, z, 0) \mathrm{d} z \mathrm{~d} y \mathrm{~d} x=-\int_{0}^{w_{x}} \int_{0}^{w_{y}} \int_{0}^{1} \psi_{i} h_{f}(x, y, z) \mathrm{d} z \mathrm{~d} y \mathrm{~d} x$

\begin{tabular}{|c|c|}
\hline \multicolumn{2}{|r|}{$\mathrm{x}$-direction } \\
\hline Eigenvalue problem & $\begin{array}{c}\frac{\mathrm{d}^{2} X_{m}}{\mathrm{~d} x^{2}}+\alpha_{m}^{2} X_{m}(x)=0 \\
\frac{\mathrm{d} X_{m}(0)}{\mathrm{d} x}-k_{1} X_{m}(0)=0 ; \frac{\mathrm{d} X_{m}\left(w_{x}\right)}{\mathrm{d} x}+k_{2} X_{m}\left(w_{x}\right)=0\end{array}$ \\
\hline Eigenquantities & $\begin{array}{c}X_{m}(x)=\alpha_{\mathrm{m}} \cos \left(\alpha_{m} x\right)+k_{1} \sin \left(\alpha_{m} x\right) ; \quad \tan \left(\alpha_{m} w_{x}\right)=\frac{\alpha_{m}\left(k_{1}+k_{2}\right)}{\left(\alpha_{m}^{2}-k_{1} k_{2}\right)}, \quad m=1,2,3, \ldots \\
\int_{0}^{w_{x}} X_{m}(x) X_{n}(x) \mathrm{d} x= \begin{cases}0, & \text { if } m \neq n \\
N x_{m}, & \text { if } m=n\end{cases} \\
N x_{m}=\int_{0}^{w_{x}} X_{m}^{2}(x) \mathrm{d} x=\frac{\alpha_{m}^{2}+k_{1}}{2}\left(w_{x}+\frac{k_{2}}{\alpha_{m}^{2}+k_{2}}\right)+\frac{k_{1}}{2}\end{array}$ \\
\hline & y-direction \\
\hline Eigenvalue problem & $\begin{array}{c}\frac{\mathrm{d}^{2} Y_{p}}{\mathrm{~d} y^{2}}+\beta_{p}^{2} Y_{p}(y)=0 \\
\frac{\mathrm{d} Y_{p}(0)}{\mathrm{d} y}=0 ; \quad \frac{\mathrm{d} Y_{p}\left(w_{y}\right)}{\mathrm{d} y}=0\end{array}$ \\
\hline Eigenquantities & $\begin{array}{r}Y_{p}(y)=\cos \left(\beta_{p} y\right) ; \quad \beta_{p}=\frac{(p-1) \pi}{w_{y}}, \quad p=1,2,3, \ldots \\
\int_{0}^{w_{y}} Y_{p}(y) Y_{q}(y) \mathrm{d} y= \begin{cases}0, & \text { if } p \neq q \\
N y_{p}, & \text { if } p=q\end{cases} \\
N y_{p}=\int_{0}^{w_{y}} Y_{p}^{2}(y) \mathrm{d} y= \begin{cases}w_{y}, & \text { if } p=1 \\
w_{y} / 2, & \text { if } p>1\end{cases} \end{array}$ \\
\hline & z-direction \\
\hline Eigenvalue problem & $\begin{array}{c}\frac{\mathrm{d}^{2} Z_{r}}{\mathrm{~d} z^{2}}+\frac{\gamma_{r}^{2}}{k_{v}} Z_{r}(z)=0 \\
\frac{\mathrm{d} Z_{r}(0)}{\mathrm{d} z}=0 ; \frac{\mathrm{d} Z_{r}(1)}{\mathrm{d} z}+\left[k^{\prime}-\frac{\sigma \gamma_{r}^{2}}{k_{v}}\right] Z_{r}(1)=0\end{array}$ \\
\hline Eigenquantities & $\begin{array}{c}Z_{r}(z)=\cos \left(\gamma_{r} z / k_{v}^{1 / 2}\right) ; \quad \tan \left(\gamma_{r} / k_{v}^{1 / 2}\right)=\sqrt{k_{v}}\left[k^{\prime}-\frac{\sigma \gamma_{r}^{2}}{k_{v}}\right] / \gamma_{r}, \quad r=1,2,3, \ldots . \\
\int_{0}^{1} Z_{r}(z) Z_{s}(z) \mathrm{d} z+\sigma Z_{r}^{2}(1)= \begin{cases}0, & \text { if } r \neq s \\
N z_{r}, & \text { if } r=s\end{cases} \\
N z_{r}=\int_{0}^{1} Z_{r}^{2}(z) \mathrm{d} z+\sigma Z_{r}^{2}(1)=\left\{\begin{array}{l}1+\sigma, \text { if } r=1 \\
\frac{1}{4}\left[2+\frac{\sqrt{k_{v}}}{\gamma_{r}} \sin \left(2 \gamma_{r} / k_{v}^{1 / 2}\right)\right]+\sigma \cos ^{2}\left(\gamma_{r} / k_{v}^{1 / 2}\right), \text { if } r>1\end{array}\right.\end{array}$ \\
\hline
\end{tabular}

Table 1. Eigenvalue problems in the $x, y$ and $z$ directions. 
After the usual manipulations, the following transformed ordinary differential system is obtained:

$$
\frac{\mathrm{d} \bar{h}_{i}(t)}{\mathrm{d} t}+\mu_{i}^{2} \bar{h}_{i}(t)=\sum_{j=1}^{\infty} A_{i j} \bar{h}_{j}(t)
$$

$$
\bar{h}_{i}(0)=\bar{f}_{i}
$$

where

$$
A_{i j}=-\sigma\left(\alpha_{m(i)}^{2}+\right.
$$$$
\left.+\beta_{p(i)}^{2}\right) \delta_{m(i) n(j)} \delta_{p(i) q(j)} Z_{r(i)}(1) Z_{s(j)}(1) / N z_{s(j)}
$$

$$
\bar{f}_{i}=-\int_{0}^{1} \tilde{\bar{Z}}_{m(i) p(i)}(z) Z_{r(i)}(z) \mathrm{d} z-\sigma \tilde{\bar{Z}}_{m(i) p(i)}(1) Z_{r(i)}(1)
$$

$\mu_{i}^{2}=\alpha_{m(i)}^{2}+\beta_{p(i)}^{2}+\gamma_{r(i)}^{2}$

and

$$
\delta_{m n}=\left\{\begin{array}{l}
0, m \neq n \\
1, m=n
\end{array} ; \quad \delta_{p q}=\left\{\begin{array}{l}
0, p \neq q \\
1, p=q
\end{array} ; \quad \delta_{r s}=\left\{\begin{array}{l}
0, r \neq s \\
1, r=s
\end{array}\right.\right.\right.
$$

The derivation of Eq. (8d) is detailed in the Appendix. The function $\tilde{\bar{Z}}_{m(i) p(i)}(z)$ is obtained from the filter solution developed in the next section.

\section{Filter problem solution}

The filter problem is also solved by GITT (Cotta, 1993), by applying a two-dimensional eigenfunction expansion, leaving the spatial variable $\mathrm{z}$ untransformed, thus recovering a decoupled ODE system in this coordinate for the transformed filter potential. The two-dimensional eigenvalue problem is obtained from the three-dimensional problem given by Eqs. (6) with solution described in Table 1, retaining only the $\mathrm{x}$ and $\mathrm{y}$ directions, and the two-dimensional eigenfunction is then given as $\phi_{i}(x, y)=X_{m}(x) Y_{p}(y)$. The two indices associated with each coordinate are then merged into a single index, as $(m, p) \rightarrow i^{*}$ or $\alpha_{m\left(i^{*}\right)}^{2}+\beta_{p\left(i^{*}\right)}^{2}=\lambda_{i^{*}}^{2}$, and the appropriate sequence combination is obtained by reordering the eigenvalues. The onedimensional Sturm-Liouville problems and respective solutions are the same presented in Table 1 for the $\mathrm{x}$ and $\mathrm{y}$-directions.

The integral transformation of problem (4) is achieved through the following integral transform pair:

$$
\begin{aligned}
& \tilde{\bar{Z}}_{i^{*}}(z)=\int_{0}^{w_{x}} \int_{0}^{w_{y}} \phi_{i^{*}}(x, y) h_{f}(x, y, z) \mathrm{d} y \mathrm{~d} x, \text { transform } \\
& h_{f}(x, y, z)=\sum_{i^{*}=1}^{\infty} \tilde{\phi}_{i^{*}}(x, y) \tilde{\bar{Z}}_{i^{*}}(z), \text { inverse }
\end{aligned}
$$

where

$$
N_{i^{*}}=N x_{m} N y_{p}, \quad \tilde{\phi}_{i^{*}}(x, y)=\phi_{i^{*}}(x, y) / N_{i^{*}}
$$

The integral transformation is accomplished through operation over Eq. (4a) and the boundary condition (4f, g) with the transformation kernel $\int_{0}^{w_{x}} \int_{0}^{w_{y}} \phi_{i^{*}}(x, y) \mathrm{d} y \mathrm{~d} x$. After the usual manipulations, the following transformed ordinary differential system is obtained:

$k_{v} \frac{\mathrm{d}^{2} \tilde{\bar{Z}}_{i^{*}}(\mathrm{z})}{\mathrm{d} z^{2}}-\lambda_{i^{*}}^{2} \tilde{\bar{Z}}_{i^{*}}(z)=\tilde{\bar{G}}_{i^{*}}$

$\frac{\mathrm{d} \tilde{\bar{Z}}_{i^{*}}(0)}{\mathrm{d} z}=0 ; \quad \frac{\mathrm{d} \tilde{\bar{Z}}_{i^{*}}(1)}{\mathrm{d} z}+k^{\prime} \tilde{\bar{Z}}_{i^{*}}(1)=0$

where

$$
\begin{aligned}
& \tilde{\bar{G}}_{i^{*}}=\int_{0}^{w_{x}} \int_{0}^{w_{y}} \phi_{i^{*}}(x, y) \delta(x-1) \delta\left(y-y_{0}\right) \mathrm{d} y \mathrm{~d} x=\phi_{i^{*}}\left(1, y_{0}\right)= \\
& =X_{m\left(i^{*}\right)}(1) Y_{p\left(i^{*}\right)}\left(y_{0}\right)
\end{aligned}
$$

The decoupled system of ordinary differential equations (Eqs. (11)) has analytical solution in the form:

$$
\tilde{\bar{Z}}_{i^{*}}(z)=\frac{\tilde{\bar{G}}_{i^{*}}}{k_{v} v_{i^{*}}^{2}}\left[\frac{k^{\prime} \cosh \left(v_{i^{*}} z\right)}{v_{i^{*}} \sinh \left(v_{i^{*}}\right)+k^{\prime} \cosh \left(v_{i^{*}}\right)}-1\right]
$$

where

$$
v_{i^{*}}=\lambda_{i^{*}} / \sqrt{k_{v}}
$$

Therefore, invoking Eq. (3) and the inverse formulas (7b) and (10b), the original problem solution is given by:

$$
h(x, y, z, t)=\sum_{i=1}^{\infty} \tilde{\Psi}_{i}(x, y, z) \bar{h}_{i}(t)+\sum_{i^{*}=1}^{\infty} \tilde{\phi}_{i^{*}}(x, y) \tilde{\bar{Z}}_{i^{*}}(z)
$$

Eqs. (8) consist of a coupled infinite system of linear differential equations for the computation of the transformed potentials, $\bar{h}_{i}(t)$. It is important to note that for $\sigma=0$ the solution for the transformed system may be analytically obtained as:

$$
\bar{h}_{i}(t)=\bar{f}_{i} \mathrm{e}^{-\mu_{i}^{2} t}
$$

For computational purposes in the general case of $\sigma \neq 0$, the expansion is then truncated to NT terms, with NT sufficiently high to ensure the targeted accuracy in each case. The analytical solution of Eqs. (8) in the general case can still be achieved by the proper algebraic matrix eigenvalue problem analysis, or alternatively, employing the matrix exponential function directly available in modern symbolic computation platforms, such as the Mathematica system (Wolfram, 2016). Here, projecting the extension of this theory to nonlinear situations such as in partially saturated porous media, a numerical approach to handle the transformed ODE system has been preferred. Several numerical methods are described in the literature for solving initial value problems such as that given by Eqs. (8). In particular, the numerical subroutine DIVPAG from the IMSL Library (2018). The DIVPAG subroutine solves an initial-value problem for ordinary differential equations using either AdamsMoulton's or Gear's BDF method. It is a well-stablished method used on the solution of stiff systems of ODEs. It is part of 
the IMSL Library written in the Fortran programming language. Such a subroutine is well-tested on the integration of this type of problem. Alternatively, the built-in routine NDSolve of the Mathematica system (Wolfram, 2016) can be considered, which was the routine employed for the case of $\sigma \neq 0$ in the present work. In light of the hybrid numericalanalytical nature of the proposed methodology, it is a perfect match to employ a mixed symbolic-numerical software system, such as Mathematica (Wolfram, 2016), for implementing the computational algorithm. First, all analytical derivations can be symbolically performed, such as the eigenvalue problem solutions, integrations, coefficients matrix assemblies, and ordinary differential equations solver automatic routines. Second, once the transformed system does not allow for an analytical solution, the numerical solver (NDSolve function) can be readily invoked in place of the symbolic one (DSolve function). It should be added that the NDSolve function automatically provides an interpolating function object that yields a continuous representation of the potentials along with the associated independent variable.

\section{Calculation of pumping rates}

The rates involved in pumping from the aquifer can be obtained by integrating Eq. (1a) over the volume $\mathrm{d} V c=\mathrm{d} z \mathrm{~d} y \mathrm{~d} x$. Based on Darcy's law, the stream depletion rate $S D R$ is the flow in the adjacent streambeds to the aquifer and can be calculated through

$$
\begin{aligned}
& S D R_{1}=-\left.\int_{0}^{w_{y}} \int_{0}^{1} \frac{\partial h(x, y, z, t)}{\partial x}\right|_{x=0} \mathrm{~d} z \mathrm{~d} y ; \\
& S D R_{2}=\left.\int_{0}^{w_{1}} \int_{0}^{1} \frac{\partial h(x, y, z, t)}{\partial x}\right|_{x=w_{x}} \mathrm{~d} z \mathrm{~d} y
\end{aligned}
$$

The pumping system of a well also depends on the contribution of other sources, namely:

1 - The Storage Release Rate $(S R R)$ is the volume of water released from the aquifer for the total volume pumped.

2 - The Leakage Rate $(L R)$, which is related to when a semiconfined aquifer is overlapped by another (separated by an aquitard with a different conductivity of the two); there is a flow of water between them, which can happen from the overlying aquifer to the underlying aquifer or vice-versa.

3 - The Gravity Drainage Rate $(G D R)$ is caused on the upper surface by decreasing the soil pressure, leading to empty release.

The integrals below define these pumping rates:

$$
\begin{aligned}
& S R R=-\int_{0}^{w_{x}} \int_{0}^{w_{y}} \int_{0}^{1} \frac{\partial h(x, y, z, t)}{\partial t} \mathrm{~d} z \mathrm{~d} y \mathrm{~d} x ; \\
& L R=\left.k_{v} \int_{0}^{w_{x}} \int_{0}^{w_{y}} \frac{\partial h(x, y, z, t)}{\partial z}\right|_{z=1} \mathrm{~d} y \mathrm{~d} x ; \\
& G D R=-\left.\sigma \int_{0}^{w_{x}} \int_{0}^{w_{y}} \frac{\partial h(x, y, z, t)}{\partial t}\right|_{z=1} \mathrm{~d} y \mathrm{~d} x
\end{aligned}
$$

In leaky aquifers, this pumping receives the contribution of the adjacent aquifer leakage rate $L R$, Eq. (15d), whereas in an unconfined aquifer, this contribution is given by the gravity drainage rate $G D R$, Eq. (15e). Therefore, the pumping rates are calculated replacing the head distribution, Eq. (13), into Eqs. (15), resulting in:

$$
\begin{aligned}
& S D R_{1}=-\sum_{i=1}^{\infty} \bar{f}_{i} \frac{B_{i} C_{i}}{N x_{m(i)} N y_{p(i)} N z_{r(i)}} \frac{\mathrm{dX}_{m(i)}(0)}{\mathrm{d} x} \bar{h}_{i}(t)- \\
& \sum_{i^{*}=1}^{\infty} \frac{B_{i^{*}}}{N x_{k\left(i^{*}\right)} N y_{l\left(i^{*}\right)}} \frac{\tilde{\bar{G}}_{i^{*}}}{\lambda_{i^{*}}^{2}}\left[\frac{k^{\prime} C_{i^{*}}}{v_{i^{*}} \sinh \left(v_{i^{*}}\right)+k^{\prime} \cosh \left(v_{i^{*}}\right)}-1\right] \frac{\mathrm{d} X_{k\left(i^{*}\right)}(0)}{\mathrm{d} x}
\end{aligned}
$$

$$
\begin{aligned}
& S D R_{2}=\sum_{i=1}^{\infty} \overline{f_{\mathrm{i}}} \frac{B_{i} C_{i}}{N x_{m(i)} N y_{p(i)} N z_{r(i)}} \frac{\mathrm{d} X_{m(i)}\left(w_{x}\right)}{\mathrm{d} x} \bar{h}_{i}(t)+ \\
& \sum_{i^{*}=1}^{\infty} \frac{B_{i^{*}}}{N x_{k\left(i^{*}\right)} N y_{1\left(i^{*}\right)}} \frac{\tilde{\bar{G}}_{i^{*}}}{\lambda_{i^{*}}^{2}}\left[\frac{k^{\prime} C_{i^{*}}}{v_{i^{*}} \sinh \left(v_{i^{*}}\right)+k^{\prime} \cosh \left(v_{i^{*}}\right)}-1\right] \frac{\mathrm{d} X_{k\left(i^{*}\right)}\left(w_{x}\right)}{\mathrm{d} x}
\end{aligned}
$$

$S R R=\sum_{i=1}^{\infty} \bar{f}_{i} \frac{B_{i} C_{i} D_{i}}{N x_{m(i)} N y_{p(i)} N z_{r(i)}} \frac{\mathrm{d} \bar{h}_{i}(t)}{\mathrm{d} t}$

$L R=k_{v} \sum_{i=1}^{\infty} \overline{f_{i}} \frac{B_{i} D_{i}}{N x_{m(i)} N y_{p(i)} N z_{r(i)}} \frac{\mathrm{d} Z_{r(i)}(1)}{\mathrm{d} z} \bar{h}_{i}(t)+$

$k_{v} \sum_{i^{*}=1}^{\infty} \frac{B_{i^{*}} D_{i^{*}} \tilde{\bar{G}}_{i^{*}}}{\lambda_{i^{*}}^{2} N x_{k\left(i^{*}\right)} N y_{1\left(i^{*}\right)}} \frac{k^{\prime} v_{i^{*}} \sinh \left(v_{i^{*}}\right)}{\left[v_{i^{*}} \sinh \left(v_{i^{*}}\right)+k^{\prime} \cosh \left(v_{i^{*}}\right)\right]}$

$G D R=-\sigma \sum_{i=1}^{\infty} \frac{B_{i} D_{i} Z_{r(i)}(1)}{N x_{m(i)} N y_{p(i)} N z_{r(i)}} \frac{\mathrm{d} \bar{h}_{i}(t)}{\mathrm{d} t}$

where

$$
\begin{aligned}
& B_{i}=\int_{0}^{w_{y}} Y_{p(i)}(y) \mathrm{d} y ; \quad C_{i}=\int_{0}^{1} Z_{r(i)}(z) \mathrm{d} z ; \quad D_{i}=\int_{0}^{w_{x}} X_{m(i)}(x) \mathrm{d} x \\
& B_{i^{*}}=\int_{0}^{w_{y}} Y_{l\left(i^{*}\right)}(y) \mathrm{d} y ; \quad C_{i^{*}}=\int_{0}^{1} \cosh \left(v_{i^{*}} z\right) \mathrm{d} z ; \quad D_{i^{*}}=\int_{0}^{w_{x}} X_{k\left(i^{*}\right)}(x) \mathrm{d} x
\end{aligned}
$$

In Eqs. (16a-e), for $\sigma=0$, the transformed potential $\bar{h}_{i}(t)$ is obtained from Eq. (14).

\section{RESULTS AND DISCUSSION}

The input parameters that were used in reporting the results are listed in Table 2. The eigenfunction expansions were truncated to a finite number of terms NT in the summations associated with the $i$ index and MT in the summations of the $i^{*}$ index. Table 3 illustrates the reordering scheme used for reducing double and triple summations into single sums for the first 20 eigenvalues.

The truncation order of MT terms for calculating the steady filter problem was set to a fixed sufficiently large value for all cases studied here, since it has little effect on the total computational cost. The coupled system of ordinary differential equations for the filtered transformed potentials, Eqs. (8), were numerically solved for variable truncation orders NT. Then, a thorough convergence analysis was performed to verify the behavior of the proposed integral transform solution. It was observed that the proposed solution can accurately reproduce the depletion rates in stream 1, including the behavior for short 
Table 2. Dimensional parameters data modified from Huang et al. (2014).

\begin{tabular}{|l|c|}
\hline Notations & Default value (unit) \\
\hline$K_{h}, K_{v}$ & $1 \mathrm{~m} /$ day, $0.1 \mathrm{~m} /$ day \\
\hline$S_{y}, S_{s}$ & $0.1,10^{-5} \mathrm{~m}^{-1}$ \\
\hline$H, W_{x}, W_{y}$ & $20 \mathrm{~m}, 500 \mathrm{~m}, 500 \mathrm{~m}$ \\
\hline$T, S$ & $20 \mathrm{~m}^{2} /$ day, $2 \times 10^{-4}$ \\
\hline$K^{\prime}, B^{\prime}$ & $0.01 \mathrm{~m} /$ day, $1 \mathrm{~m}$ \\
\hline$K_{l}, B_{1}$ & $1 \mathrm{~m} /$ day, $1 \mathrm{~m}$ \\
\hline$K_{2}, B_{2}$ & $1 \mathrm{~m} /$ day, $1 \mathrm{~m}$ \\
\hline$x_{0}^{*}, y_{0}^{*}$ & $50 \mathrm{~m}, 250 \mathrm{~m}$ \\
\hline$Q$ & none \\
\hline
\end{tabular}

Table 3. Eigenvalues reordering scheme for the double and triple eigenfunction expansions $\left(k_{2}=0\right.$ and $\left.w_{x}=10\right)$.

\begin{tabular}{|c|c|c|c|c|c|c|c|c|}
\hline \multicolumn{4}{|c|}{$\begin{array}{c}\text { Double summation } \\
\text { reordering }\end{array}$} & \multicolumn{5}{|c|}{ Triple summation } \\
\hline$m$ & $p$ & $i^{*}$ & $\lambda_{i}{ }^{*}$ & $\mathrm{~m}$ & $\mathrm{p}$ & $\mathrm{r}$ & $i$ & $\mu_{i}$ \\
\hline 1 & 1 & 1 & $\mathbf{0 . 1 5 6 7 6 6}$ & 1 & 1 & 1 & 1 & $\mathbf{0 . 1 5 6 7 6 6}$ \\
\hline 1 & 2 & 2 & $\mathbf{0 . 3 5 1 1 0 1}$ & 1 & 2 & 1 & 2 & $\mathbf{0 . 3 5 1 1 0 1}$ \\
\hline 2 & 1 & 3 & $\mathbf{0 . 4 7 0 2 9 8}$ & 2 & 1 & 1 & 3 & $\mathbf{0 . 4 7 0 2 9 8}$ \\
\hline 2 & 2 & 4 & $\mathbf{0 . 5 6 5 5 7 6}$ & 2 & 2 & 1 & 4 & $\mathbf{0 . 5 6 5 5 7 6}$ \\
\hline 1 & 3 & 5 & $\mathbf{0 . 6 4 7 5 8 0}$ & 1 & 3 & 1 & 5 & $\mathbf{0 . 6 4 7 5 8 0}$ \\
\hline 3 & 1 & 6 & $\mathbf{0 . 7 8 3 8 3 1}$ & 3 & 1 & 1 & 6 & $\mathbf{0 . 7 8 3 8 3 1}$ \\
\hline 2 & 3 & 7 & $\mathbf{0 . 7 8 4 8 3 4}$ & 2 & 3 & 1 & 7 & $\mathbf{0 . 7 8 4 8 3 4}$ \\
\hline 3 & 2 & 8 & $\mathbf{0 . 8 4 4 4 4 4}$ & 3 & 2 & 1 & 8 & $\mathbf{0 . 8 4 4 4 4 4}$ \\
\hline 1 & 4 & 9 & $\mathbf{0 . 9 5 5 4 2 7}$ & 1 & 4 & 1 & 9 & $\mathbf{0 . 9 5 5 4 2 7}$ \\
\hline 3 & 3 & 10 & $\mathbf{1 . 0 0 4 5 7 7}$ & 3 & 3 & 1 & 10 & $\mathbf{1 . 0 0 4 5 7 7}$ \\
\hline 2 & 4 & 11 & $\mathbf{1 . 0 5 3 3 0 2}$ & 2 & 4 & 1 & 11 & $\mathbf{1 . 0 5 3 3 0 2}$ \\
\hline 4 & 1 & 12 & $\mathbf{1 . 0 9 7 3 6 3}$ & 4 & 1 & 1 & 12 & $\mathbf{1 . 0 9 7 3 6 3}$ \\
\hline 4 & 2 & 13 & $\mathbf{1 . 1 4 1 4 4 7}$ & 4 & 2 & 1 & 13 & $\mathbf{1 . 1 4 1 4 4 7}$ \\
\hline 3 & 4 & 14 & $\mathbf{1 . 2 2 5 8 2 8}$ & 3 & 4 & 1 & 14 & $\mathbf{1 . 2 2 5 8 2 8}$ \\
\hline 4 & 3 & 15 & $\mathbf{1 . 2 6 4 5 1 2}$ & 1 & 1 & 2 & 15 & $\mathbf{1 . 2 5 2 6 7 7}$ \\
\hline 1 & 5 & 16 & $\mathbf{1 . 2 6 6 3 7 8}$ & 4 & 3 & 1 & 16 & $\mathbf{1 . 2 6 4 5 1 2}$ \\
\hline 2 & 5 & 17 & $\mathbf{1 . 3 4 1 7 5 9}$ & 1 & 5 & 1 & 17 & $\mathbf{1 . 2 6 6 3 7 8}$ \\
\hline 5 & 1 & 18 & $\mathbf{1 . 4 1 0 8 9 6}$ & 1 & 2 & 2 & 18 & $\mathbf{1 . 2 9 1 4 7 1}$ \\
\hline 5 & 2 & 19 & $\mathbf{1 . 4 4 5 4 4 9}$ & 2 & 1 & 2 & 19 & $\mathbf{1 . 3 2 8 8 3 6}$ \\
\hline 4 & 4 & 20 & $\mathbf{1 . 4 4 6 5 3 7}$ & 2 & 5 & 1 & 20 & $\mathbf{1 . 3 4 1 7 5 9}$ \\
\hline
\end{tabular}

periods, as can be seen in Table 4. Convergence analysis of the $S D R_{1}$ for $k_{2}=0$ and $w_{x}=10$ (unconfined aquifer) is briefly illustrated in Table 4, at different dimensionless times t, varying the number of terms in the expansion, NT. Note that full convergence to three significant digits, even at the shortest time $t=$ 20 or $t^{*}=0.5$ day, is obtained for NT always less than 1000 , with a marked improvement of convergence rate for larger values of $t$. The complete set of dimensionless parameters employed in this simulation is: $w_{x}=w_{y}=10, k_{1}=50, k_{2}=0, k_{v}=$ $0.625, k^{\prime}=5, \sigma=500, x_{0}=1, y_{0}=5$.

Figure 2 shows the $S D R_{1}$ evolution for the confined, leaky and unconfined aquifer cases obtained through Eq. (16a). Previous approaches obtain analytical solution only confined case, $\sigma=0$ and $k^{\prime}=0$. However, the present work proposed an analytical solution which include the leakage aquifer case, $\sigma=0$ and $k^{\prime} \neq 0$. The transformed potential $\bar{h}_{i}(t)$ is only numerically obtained from Eqs. (8) for unconfined case $\sigma \neq 0$. The transformed potentials are obtained from the numerical solution of the system of ODEs given in Eq. (8a), subject to the transformed initial conditions given in Eq. (8b). The present results show excellent agreement with previously reported approximate analytical solutions (Huang et al., 2014). Figure 3 shows

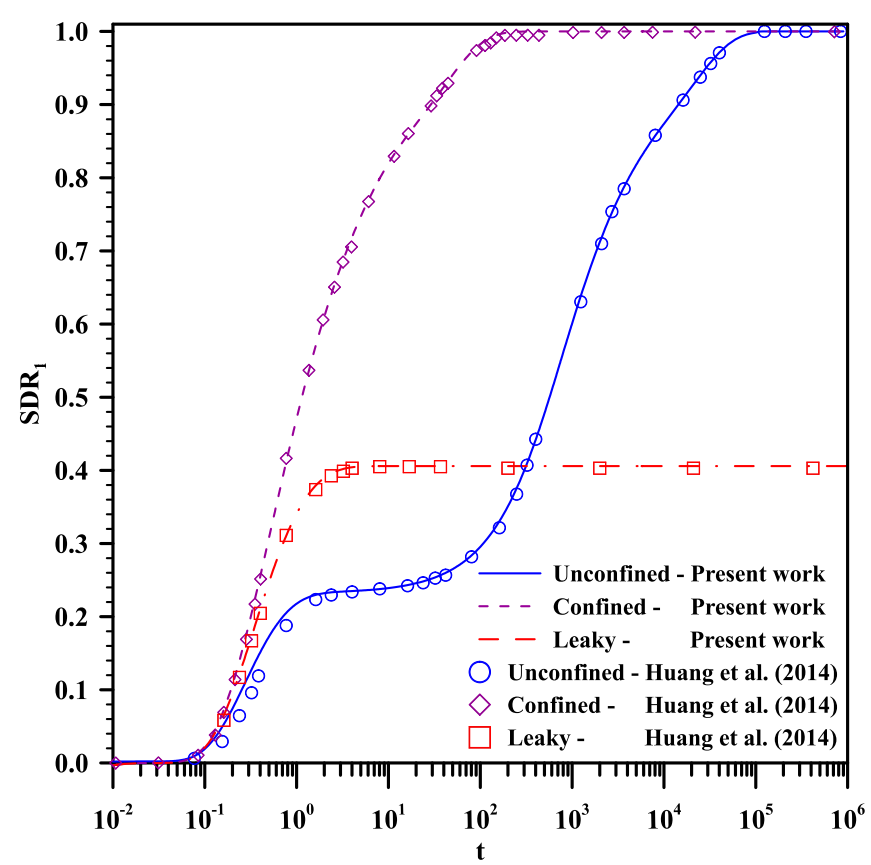

Fig. 2. $S D R_{1}$ distribution for confined, leaky and unconfined aquifers.

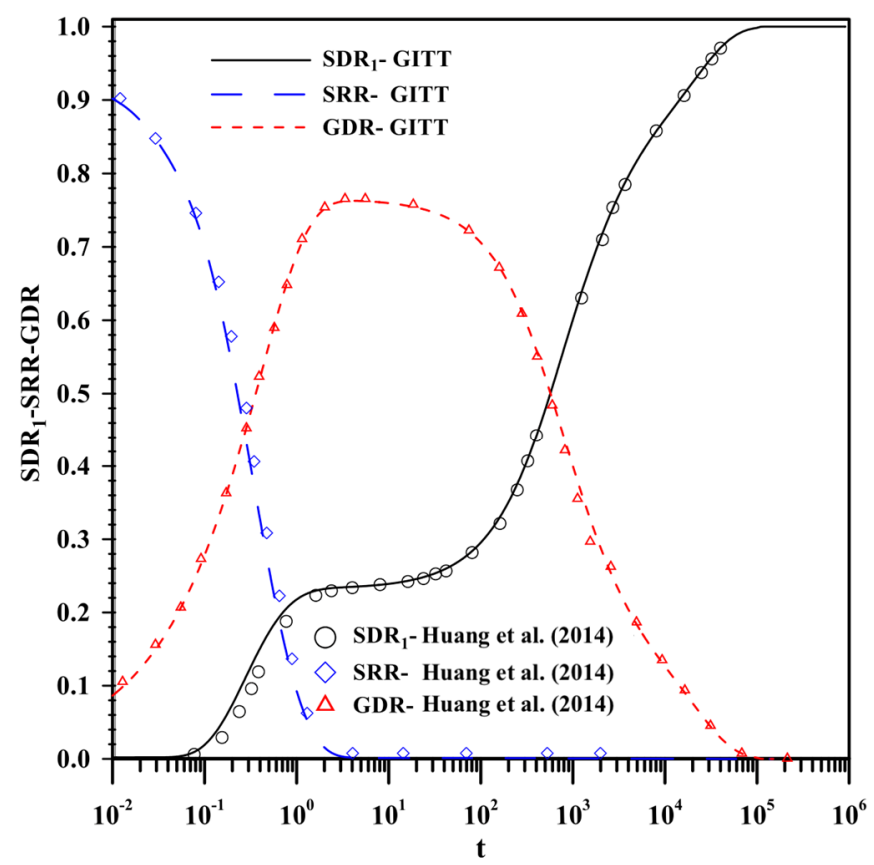

Fig. 3. $S D R_{1}, S R R$ and $G D R$ distributions for an unconfined aquifer.

the rates $S D R_{1}, S R R$ and $G D R$ for $k^{\prime}=0$ and $\sigma \neq 0$ as obtained from Eqs. (16a), (16c) and (16e), respectively. Again, it is verified an excellent agreement between the present results shown in Figure 3 with those of reference works. Here, it is essential to mention that for unconfined aquifers, at any time, the sum of the $G D R, S R R$, and $S D R$ is equal to unity. Therefore, in Fig. 3, we observe $S R R$ beginning to decrease from a value near one until it reaches a zero value approximately in the interval $1 \leq t \leq 100$, and the $G D R$ reaches its maximum. This location is precisely the first mode place for the $S D R$. As both $S R R$ and $G D R$ decline after this interval, the $S D R$ continues to increase until the second mode occurs when the pumping reaches a steady state.

Figure 4 shows the effect of varying the distance between streams 1 and 2, with $k_{2} \neq 0$, on the time variation of $S D R_{1}$ and $S D R_{2}$, where it can be noted how the $S D R$ is affected by the 


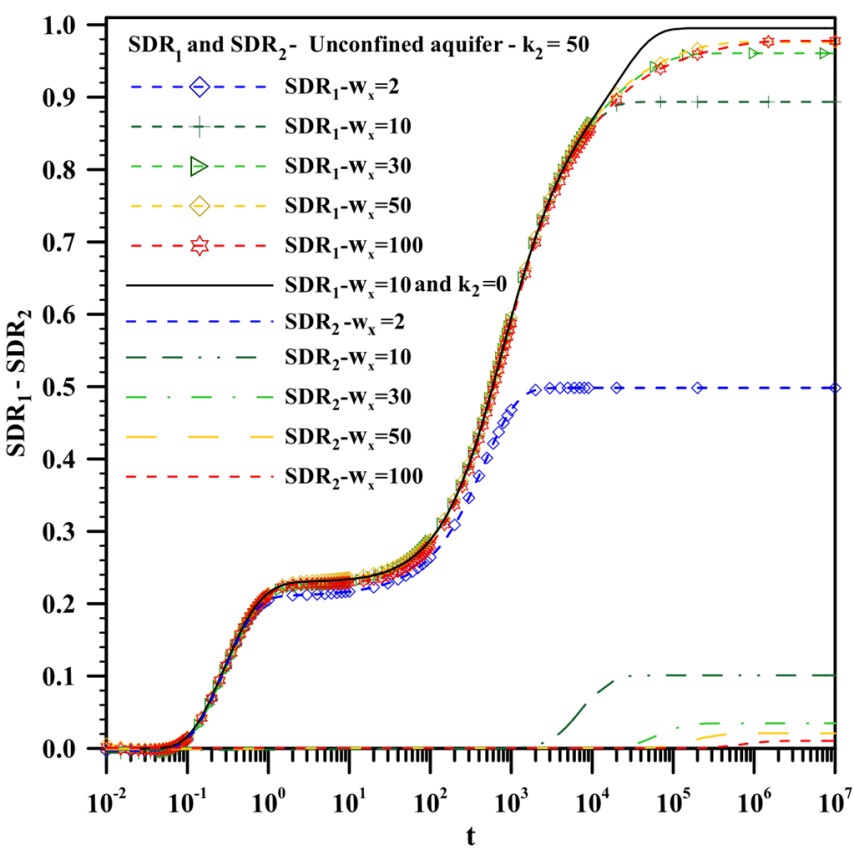

Fig. 4. $S D R_{1}$ and $S D R_{2}$ distributions for an unconfined aquifer varying $w_{x}$ and for $k_{1}=k_{2}=50$.

distance between the well and the streambed. Miller et al. (2007) discussed the relationship between the depletion rate and the distance between the well and the streams, showing that there is an inverse relationship between them, i.e., $S D R$ increases faster when $w_{x}$ decreases. It is worth noting that the increase in distance between streams makes the depletion rate tend to resemble the non-flow case in streambed 2 or $S D R_{2} \rightarrow 0$ when $w_{x}>50$ (or $W_{x}>2500$ meters for the employed parameters). As can be seen in Figs. 2-4 the stream depletion rate reaches steady state when $t>10^{5}$ and the head distribution remains constant from this moment, since from this point on the well pumping has contribution only from $S D R_{1}$ and $S D R_{2}$. In Fig. 4 we may also note that increasing the river distances, keeping the bed position fixed, for $w_{x}>10\left(W_{x}>500 \mathrm{~m}\right)$, the $S R D$ in the streambed two is only started after approximated $t>10^{3}\left(t^{*}>25\right.$ days). Increasing $w_{x}$ to $30\left(W_{x}>1500 \mathrm{~m}\right.$, i.e., the streambed two is $1450 \mathrm{~m}$ far from the well), the $S D R$ is started only when $t>$ $10^{4}\left(t^{*}>250\right.$ days $)$.

Figure 5 shows the dimensionless head profiles for an unconfined aquifer in the $\mathrm{x}$-direction at $y=y_{0}$, with $k_{2}=0$ and $w_{x}$ $=10$. Fig. 5a shows a head variation profile varying in time for a constant specific yield, $S_{y}=0.1$, whereas Fig. $5 \mathrm{~b}$ shows the head profile for fixed time $t=10^{3}$, with $S_{y}$ ranging from 0.001 to 0.3 . We noted that the increase of the time, $t>10^{3}$, and decrease in the specific yield, $S_{y}<0.1$, implies the confinement of the aquifer. Based on Fig. 3, near $t=10^{3}$, the $G D R=S D R$ and after they tend to zero while $S D R$ changes to behave like a confined aquifer. The recovery reduction of the aquifer can


Fig. 5. Dimensionless head profiles for an unconfined aquifer as functions of the x-direction at $y=y_{0}$ and $z=1$ with $k_{2}=0$ and $w_{x}=$ 10 for: (a) different times with $S_{y}=0.1$; (b) for different specific yields $S_{y}$ with $t=10^{3}$.

explain this behavior due to the action of gravity, which would represent the reduction in its recharging capacity. As shown in Fig. 5, this occurs immediately, decreasing the specific yield or prolonging pumping times.

Table 4. Convergence behavior of the $S D R_{1}$ for $k_{2}=0$ and $w_{x}=10$ for the case of an unconfined aquifer.

\begin{tabular}{|c|c|c|c|c|c|c|c|c|c|}
\hline $\boldsymbol{t}$ & $\boldsymbol{t}^{*}(\mathbf{d a y s})$ & $\boldsymbol{N T}=\mathbf{3 0 0}$ & $\boldsymbol{N T}=\mathbf{4 0 0}$ & $\boldsymbol{N T}=\mathbf{5 0 0}$ & $\boldsymbol{N T}=\mathbf{6 0 0}$ & $\boldsymbol{N T}=\mathbf{7 0 0}$ & $\boldsymbol{N T}=\mathbf{8 0 0}$ & $\boldsymbol{N T}=\mathbf{9 0 0}$ & $\boldsymbol{N T}=\mathbf{1 0 0 0}$ \\
\hline $\mathbf{2 0}$ & $\mathbf{0 . 5}$ & 0.215 & 0.247 & 0.244 & 0.247 & 0.250 & 0.251 & 0.248 & 0.248 \\
\hline $\mathbf{5 0}$ & $\mathbf{1 . 2 5}$ & 0.237 & 0.252 & 0.259 & 0.261 & 0.263 & 0.263 & 0.263 & 0.263 \\
\hline $\mathbf{1 0 0}$ & $\mathbf{2 . 5}$ & 0.272 & 0.279 & 0.285 & 0.287 & 0.288 & 0.288 & 0.289 & 0.289 \\
\hline $\mathbf{5 0 0}$ & $\mathbf{1 2 . 5}$ & 0.468 & 0.468 & 0.468 & 0.468 & 0.468 & 0.468 & 0.468 & 0.468 \\
\hline $\mathbf{1 0 0 0}$ & $\mathbf{2 5}$ & 0.592 & 0.592 & 0.592 & 0.592 & 0.592 & 0.592 & 0.592 & 0.592 \\
\hline $\mathbf{5 0 0 0}$ & $\mathbf{1 2 5}$ & 0.810 & 0.810 & 0.810 & 0.810 & 0.810 & 0.810 & 0.810 & 0.810 \\
\hline
\end{tabular}



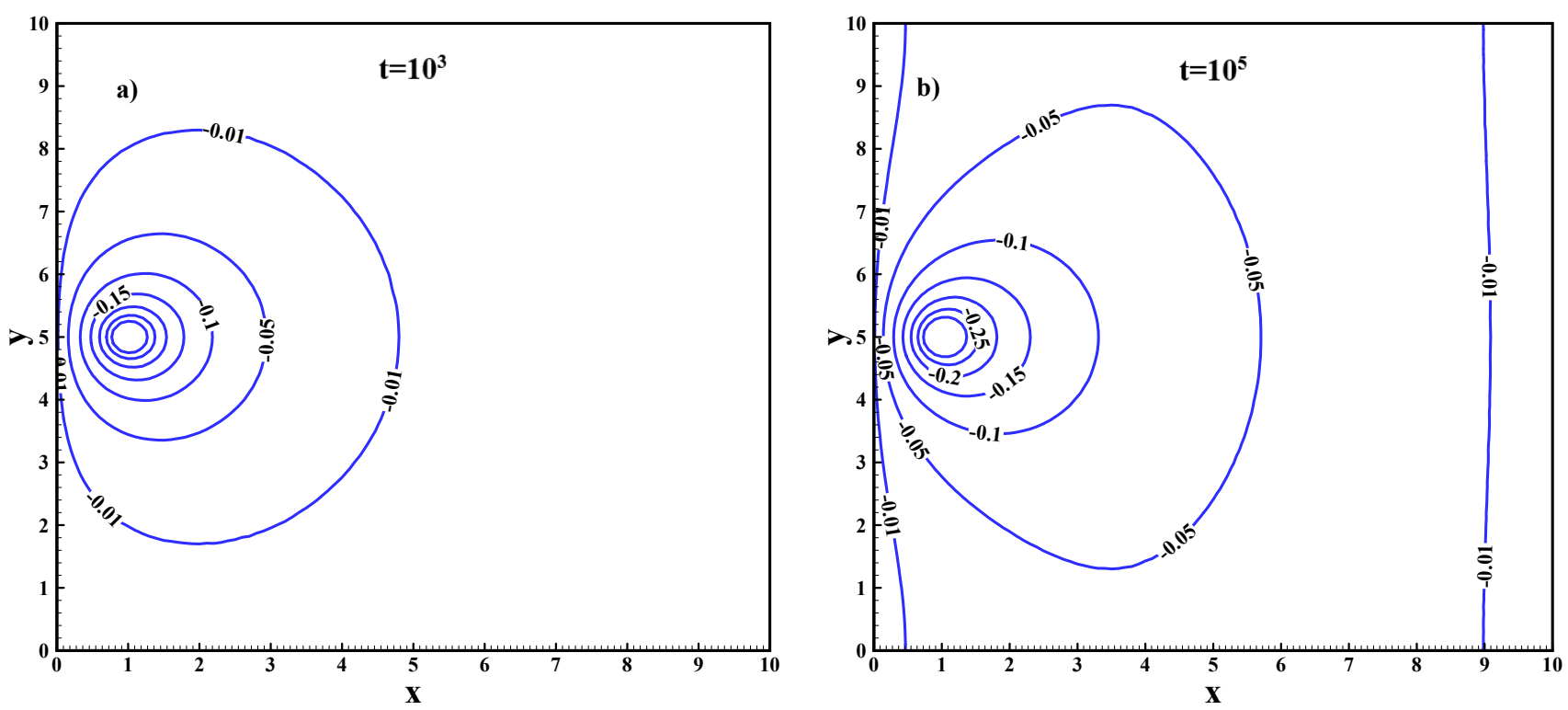

Fig. 6. Dimensionless head profiles at $z=0$ for $k_{1}=k_{2}=50$ and $w_{x}=10$ : (a) $t=10^{3}$; (b) $t=10^{5}$.
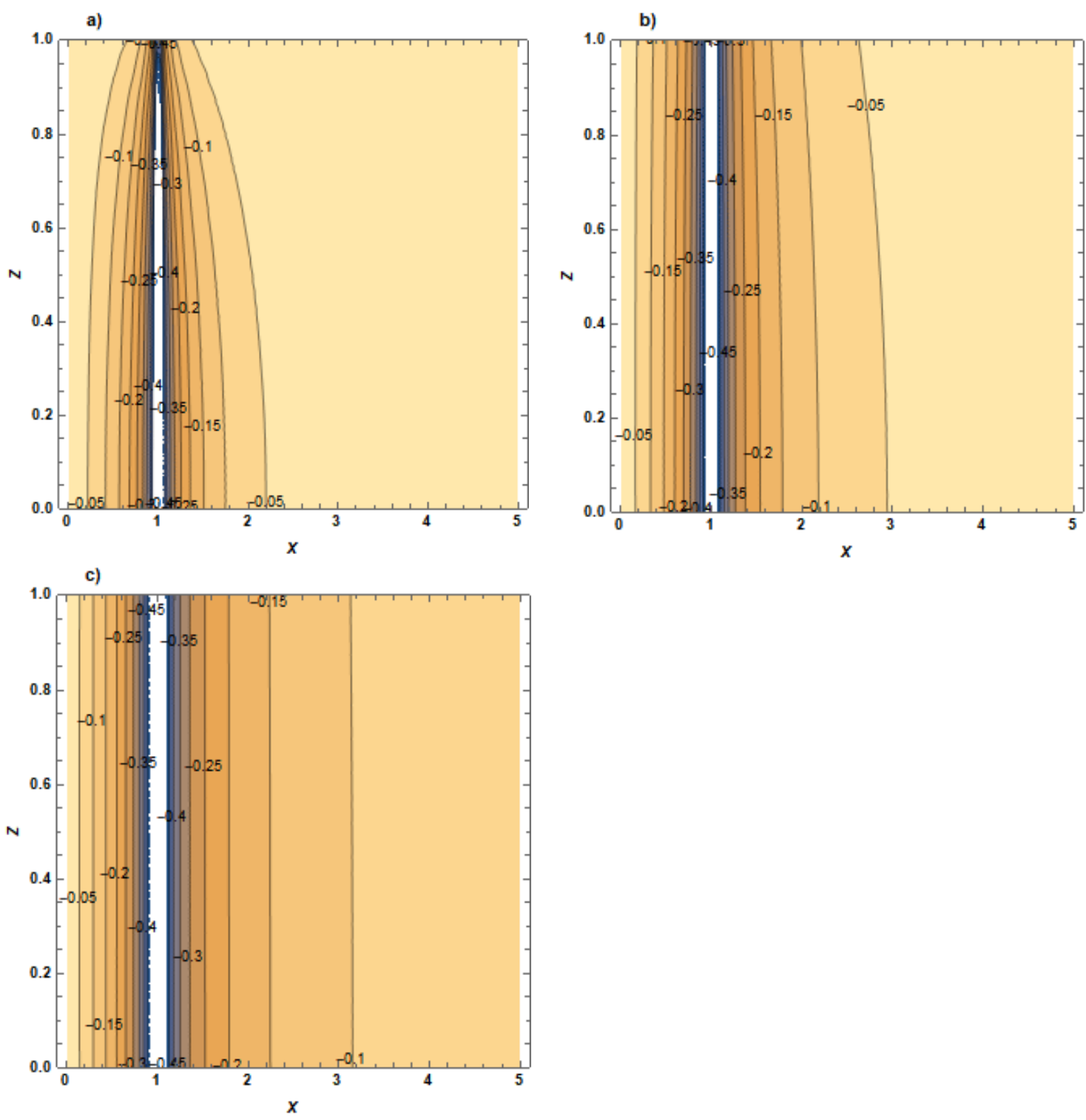

Fig. 7. Dimensionless head profiles for an unconfined aquifer in the z-direction at $y=y_{0}$ for $x \in\left[0, w_{x} / 2\right]$ for different times: (a) $t=10^{2}$; (b) $t=10^{3}$, (c) $t=10^{4}$. 
Since the source term representing the vertical well is the Dirac delta equation, it consists of an infinite pulse located at $\left(x_{0}, y_{0}\right)$. Depending on its intensity, it acts with equal distribution throughout the domain in the $\mathrm{z}$-direction. For this reason, the pressures $\mathrm{h}$ in the $\mathrm{z}$-direction vary only in the initial pumping times and become constant for long times, changing only in the other directions involved, as shown in Figs. 6 and 7. In Figure 6, we note that the variable horizontal pressure behavior in anisotropic medium, and we can identify the expansion of the drawdown cone varying in space. Fig 7 demonstrates that the pressure in the $\mathrm{z}$-direction ceases to exert influence on the system for $t \geq 10^{3}$.

\section{CONCLUDING REMARKS}

The present work proposes analytical and hybrid (numericalanalytical) solutions through the Generalized Integral Transform Technique (GITT) to obtain the transient threedimensional hydrological behavior of a limited aquifer between two parallel streams. A semipermeable streambed defined by boundary conditions of the third type and no flow condition in the direction parallel to the streams is considered, that is drained by a vertical, fully penetrating well and with an upper boundary condition that models so much a confined, leaky, or unconfined aquifer. For accelerate the convergence of this eigenfunction expansion solution, a steady-state filter was used to eliminate the source term in the general equation of the head, associated with the well. The filter problem solution was obtained through integral transforms as well, considering a twodimensional eigenvalue problem as the base of the eigenfunction expansion. The filtered head solution formulated as a homogeneous problem can then be explicitly and analytically obtained for $\sigma=0$, while a numerical-analytical solution is achieved when $\sigma \neq 0$. Previous works have achieved analytical solutions only for the confined aquifer situation. Although the GITT is a hybrid method, it automatically recovers fully analytical solutions once the appropriate eigenfunction expansion base is employed, such as here proposed for the case of a leaking aquifer, while obtaining an analytical-numerical solution only the more involved case of a free aquifer. To reduce computational costs, a reordering scheme for the eigenvalues involved in the filter and filtered homogeneous problems was employed. In addition to reducing the sums, the reordering speeds up convergence, and the program developed to calculate the eigenvalues orders them from the smallest (most important) to the largest (least important). Since the solution is obtained by a series governed by an exponential, these eigenvalues lose influence when they assume exceptionally large values which resulted in a marked reduction of the number of terms NT necessary for convergence. The computed results have shown that the proposed solution offers fully converged flow rates and head distributions, even close to the well, at modest computation effort. The verification with previously reported simulations provided additional confidence in the GITT solutions, offering the possibility for a brief analysis on the variation of other parameters of physical interest or different mediums.

The results obtained in the present work show that GITT (widely used in heat transfer problems) can be very well applied to fluid flow problems in saturated or even unsaturated porous media. More complex models, including irregular geometries, can be obtained by combining previous advancements in the same methodology.

Acknowledgements. The authors would like to acknowledge the financial support provided by the Brazilian sponsoring agencies
CNPq and CAPES. One of the authors (EMS) would like to acknowledge the collaboration of the Scientific Computing Laboratory of the Universidade Federal do Sul e Sudeste do Pará (LCC/CTIC/UNIFESSPA).

\section{REFERENCES}

Almeida, A.R., Cotta, R.M., 1995. Integral transform methodology for convection-diffusion problems in petroleum reservoir engineering. International Journal of Heat and Mass Transfer, 38, 3359-3367.

Almeida, A.R., Cotta, R.M., 1996a. Analytical solution of the tracer equation for the homogeneous five-spot problem. SPE Journal, 1, 31-38.

Almeida, A.R., Cotta, R.M., 1996b. A comparison of convergence acceleration schemes for eigenfunction expansions of partial differential equations. International Journal of $\mathrm{Nu}$ merical Methods for Heat \& Fluid Flow, 6, 85-97.

Bakker, M., 2004. Transient analytic elements for periodic Dupuit-Forchheimer flow. Advances in Water Resources, 27, 3-12.

Bakker, M., Strack, O.D.L., 2003. Analytic elements for multiaquifer flow. Journal of Hydrology, 271, 119-129.

Bakker, M., Kelson, V.A., Luther, K.H., 2005. Multilayer analytic element modeling of radial collector wells. Groundwater, 43, 926-934.

Barros, F.P.J., Mills, W.B., Cotta, R.M., 2006. Integral transform solution of two-dimensional model for contaminants dispersion in rivers and channels with spatially variable coefficients. Environmental Modelling and Software, 21, 699-709.

Barros, F.P.J., Cotta, R.M., 2007. Integral transforms for threedimensional steady turbulent dispersion in rivers and channels. Applied Mathematical Modelling, 31, 2719-2732.

Butler, J.J., Zlotnik, B.A., Tsou, M.S., 2001. Drawdown and stream depletion produced by pumping in the vicinity of a partially penetrating stream. Groundwater, 39, 651-659.

Butler, J.J., Zhan, X., Zlotnik, V.A., 2007. Pumping-induced drawdown and stream depletion in a leaky aquifer system. Groundwater, 45, 178-186.

Chen, C., Wan, J., Zhan, H., 2003. Theoretical and experimental studies of coupled seepage-pipe flow to a horizontal well. Journal of Hydrology, 281, 159-171.

Correa, E.J., Cotta, R.M., Orlande, H.R.B., 1997. On the reduction of computational costs in eigenfunction expansions of multidimensional diffusion problems. International Journal of Numerical Methods for Heat \& Fluid Flow, 7, 675-695.

Cotta, R.M., 1990. Hybrid numerical-analytical approach to nonlinear diffusion problems. Numerical Heat Transfer, Part B Fundamentals, 127, 217-226.

Cotta, R.M., 1993. Integral Transforms in Computational Heat and Fluid Flow. CRC Press, Boca Raton.

Cotta, R.M., 1994. Benchmark results in computational heat and fluid flow: - the integral transform method. International Journal of Heat and Mass Transfer (invited paper), 37, 381394.

Cotta, R.M., 1998. The Integral Transform Method in Thermal and Fluids Sciences and Engineering. Begell House, New York.

Cotta, R.M., Mikhailov, M.D., 1997. Heat Conduction: Lumped Analysis, Integral Transforms, Symbolic Computation. Wiley-Interscience, Chichester.

Cotta, R.M, Mikhailov, M.D., 2006. Hybrid methods and symbolic computations. In: Minkowycz, W.J., Sparrow, E.M., Murthy, J.Y. (Eds.): Handbook of Numerical Heat Transfer. 2nd ed. Wiley, New York, pp. 493-522. 
Cotta, R.M., Ungs, M.J., Mikhailov, M.D., 2003. Contaminant transport in finite fractured porous medium: integral transforms and lumped-differential formulations. Annals of $\mathrm{Nu}-$ clear Energy, 30, 261-285.

Cotta, R.M., Knupp, D.C., Naveira-Cotta, C.P., Sphaier, L.A., Quaresma, J.N.N., 2013. Unified integral transforms algorithm for solving multidimensional nonlinear convectiondiffusion problems. Numerical Heat Transfer, Part A Applications, 63, 840-866.

Cotta, R.M., Knupp, D.C., Naveira-Cotta, C.P., Sphaier, L.A., Quaresma, J.N.N., 2014. The unified integral transforms (UNIT) algorithm with total and partial transformation. Computational Thermal Sciences, 6, 507-524.

Cotta, R.M., Knupp, D.C., Quaresma J.N.N., 2018a. Analytical methods in heat transfer. In: Kulacki, F.A. et al. (Eds.): Handbook of Thermal Science and Engineering, Chapter 1. Springer International Publishing.

Cotta, R.M., Naveira-Cotta, C.P., Knupp, D.C., Zotin, J.L.Z., Pontes, P.C., Almeida, A.P., 2018b. Recent advances in computational-analytical integral transforms for convectiondiffusion problems. Heat and Mass Transfer (invited paper), 54, 2475-2496.

Cotta, R.M., Su, J., Pontedeiro, A.C., Lisboa, K.M., 2018c. Computational-analytical integral transforms and lumpeddifferential formulations: benchmarks and applications in nuclear technology. Special Lecture at the 9th Int. Symp. on Turbulence, Heat and Mass Transfer, Rio de Janeiro. In: Silva Freire, A.P. et al. (Eds.): Turbulence, Heat and Mass Transfer 9, Begell House, New York, pp. 129-144.

Glover, R.E., Balmer, G.G., 1954. River depletion resulting from pumping a well near a river. Transactions of the American Geophysical Union, 35, 468-470.

Hantush, M.S., 1965. Wells near streams with semi-pervious beds. Journal of Geophysical Research, 70, 2829-2838.

Hantush, M.S., Papadopoulos, I.S., 1962. Flow of groundwater to collector wells. Journal of the Hydraulics Division, 88, 221-244.

Huang, C.S., Chen, Y.L., Yeh, H.D., 2011. A general analytical solution for flow to a single horizontal well by Fourier and Laplace transforms. Advances in Water Resources, 34, 640 648.

Huang, C.S., Tsou, P.R., Yeh, H.D., 2012a. An analytical solution for a radial collector well near a stream with a lowpermeability streambed. Journal of Hydrology, 446, 48-58.

Huang, C.S., Yeh, H.D., Chang, C.H., 2012b. A general analytical solution for groundwater fluctuations due to dual tide in long but narrow islands. Water Resources Research, 48, W05508.

Huang, C.S., Lin, W.S, Yeh, H.D., 2014. Stream filtration induced by pumping in a confined, unconfined or leaky aquifer bounded by two parallel streams or by a stream and an impervious stratum. Journal of Hydrology, 513, 28-44.

Huang, C.S., Chen, J.J., Yeh, H.D., 2016. Approximate analysis of three-dimensional groundwater flow toward a radial collector well in a finite-extent unconfined aquifer. Hydrology and Earth System Sciences, 20, 55-71.

Hunt, B., 1999. Unsteady stream depletion from ground water pumping. Groundwater, 37, 98-102.

Hunt, B., 2009. Stream depletion in a two-layer leaky aquifer system. Journal of Hydrologic Engineering, 14, 895-903.

Hunt, B., Weir, J., Clausen, B., 2001. A stream depletion field experiment. Groundwater, 39, 283-289.

Intaraprasong, T., Zhan, H., 2007. Capture zone between two streams. Journal of Hydrology, 338, 297-307.

IMSL® Fortran Numerical Library, 2018. Version 2018, Rogue
Wave Software Inc., Boulder, USA. https://help.imsl.com/ fortran/current/pdf/FortranFC-v2020.0.pdf

Knowling, M.J., Werner, A.D., 2016. Estimability of recharge through groundwater model calibration: Insights from a field-scale steady-state example. Journal of Hydrology, 540, 973-987.

Liu, C., Szecsody, J.E., Zachara, J.M., Ball, W.P., 2000. Use of the generalized integral transform method for solving equations of solute transport in porous media. Advances in Water Resources, 23, 483-492.

Loizeau, S., Rossier, Y., Gaudet, J.P., Refloch, A., Besnard, K., Angulo-Jaramillo, R., Lassabatere, L., 2017. Water infiltration in an aquifer recharge basin affected by temperature and air entrapment. Journal of Hydrology and Hydromechanics, $65,222-233$.

Malama, B., Kuhlman K.L., Barrash, W., 2007. Semi-analytical solution for flow in leaky unconfined aquifer-aquitard systems. Journal of Hydrology, 346, 59-68.

Mikhailov, M.D., Cotta, R.M., 1996. Ordering rules for double and triple eigenseries in the solution of multidimensional heat and fluid flow problems. International Communications in Heat and Mass Transfer, 23, 299-303.

Mikhailov, M.D., Özisik, M.N., 1984. Unified Analysis and Solutions of Heat and Mass Diffusion. John Wiley, New York.

Mikhailov, M.D., Özisik, M.N., 1985. Unified solutions of heat diffusion in a finite region involving a surface film of finite heat capacity. International Journal of Heat and Mass Transfer, 28, 1039-1045.

Miller, C.D., Durnford, D., Halstead, M.R., Altenhofen, J., Flory, V., 2007. Stream depletion in alluvial valleys using the SDF semianalytical model. Groundwater, 45, 506-514.

Naveira-Cotta, C.P., Pontedeiro, E.M., Cotta, R.M., Su, J., van Genuchten, M.Th., 2013. Environmental impact assessment of liquid waste ponds in uranium milling installations. Waste and Biomass Valorization, 4, 197-211.

Özisik, M.N., 1980. Heat Conduction. John Wiley \& Sons, Inc., New York.

Pastore, N., Cherubini, C., Giasi, C.I., 2017. Kinematic diffusion approach to describe recharge phenomena in unsaturated fractured chalk. Journal of Hydrology and Hydromechanics, 65, 287-296.

Santos, C.A.C., Quaresma, J.N.N., Lima, J.A., 2001. Benchmark Results for Convective Heat Transfer in Ducts: - The Integral Transform Approach. E-Papers, Rio de Janeiro.

Strack, O.D.L., 1999. Principles of the analytic element method. Journal of Hydrology, 226, 128-138.

Strack, O.D.L., 2003. Theory and applications of the analytic element method. Reviews of Geophysics, 41, 1-16.

Sun, D., Zhan, H., 2006. Flow to a horizontal well in an aquitard-aquifer system. Journal of Hydrology, 321, 364376.

Sun, D., Zhan, H., 2007. Pumping induced depletion from two streams. Advances in Water Resources, 30, 1016-1026.

Theis, C.V., 1941. The effect of a well on the flow of a nearby stream. Transactions of the American Geophysical Union, 22, 734-738.

Ward, N.D., Lough, H., 2011. Stream depletion from pumping a semiconfined aquifer in a two-layer leaky aquifer system. Journal of Hydrologic Engineering, 16, 955-959.

Wolfram, S., 2016. Mathematica v. 10. Wolfram Research Inc.

Zhan, H., Park, E., 2003. Horizontal well hydraulics in leaky aquifers. Journal of Hydrology, 281, 129-146.

Zhan, H., Zlotnik, V.A., 2002. Ground water flow to horizontal and slanted wells in unconfined aquifers. Water Resources Research, 38, WR000401. 


\section{APPENDIX: Integral transformation of the initial condition}

The integral transformation of the initial condition given by Eq. (5b) is given by:

$\int_{0}^{w_{x}} \int_{0}^{w_{y}} \int_{0}^{1} \psi_{i}(x, y, z) h_{H}(x, y, z, 0) \mathrm{d} z \mathrm{~d} y \mathrm{~d} x=$
$-\int_{0}^{w_{x}} \int_{0}^{w_{y}} \int_{0}^{1} \psi_{i}(x, y, z) h_{f}(x, y, z) \mathrm{d} z \mathrm{~d} y \mathrm{~d} x$

From Eq. (7a), it follows that:

$\bar{h}_{m p r}(0)-\sigma \int_{0}^{w_{x}} \int_{0}^{w_{y}} \psi_{i}(x, y, 1) h_{H}(x, y, 1,0) \mathrm{d} y \mathrm{~d} x=$

$-\int_{0}^{w_{x}} \int_{0}^{w_{y}} \int_{0}^{1} \psi_{i}(x, y, z) h_{f}(x, y, z) \mathrm{d} z \mathrm{~d} y \mathrm{~d} x$

However, from Eq. (5b), $h_{H}(x, y, 1,0)=-h_{f}(x, y, 1)$, then:

$\bar{h}_{m p r}(0)=-\int_{0}^{w_{x}} \int_{0}^{w_{y}} \int_{0}^{1} \psi_{i}(x, y, z) h_{f}(x, y, z) \mathrm{d} z \mathrm{~d} y \mathrm{~d} x$

$-\sigma \int_{0}^{w_{x}} \int_{0}^{w_{y}} \psi_{i}(x, y, 1) h_{f}(x, y, 1) \mathrm{d} y \mathrm{~d} x$

Replacing in Eq. (A3) the head filter $h_{f}(x, y, z)$, given by Eq. (12), into Eq. (7b) and the eigenfunctions expression, $\psi_{i}(x, y, z)$ $=X_{m}(x) Y_{p}(y) Z_{r}(z)$, it results

$\bar{h}_{m p r}(0)=-\sum_{n=1}^{\infty} \sum_{q=1}^{\infty} \int_{0}^{w_{x}} \int_{0}^{w_{y}} \frac{X_{m}(x) X_{n}(x) Y_{p}(y) Y_{q}(y)}{N x_{n} N y_{q}} \mathrm{~d} y \mathrm{~d} x \int_{0}^{1} Z_{r}(z) \tilde{\bar{Z}}_{n q}(z) \mathrm{d} z-$ $\sigma \sum_{n=1}^{\infty} \sum_{q=1}^{\infty} \int_{0}^{w_{x}} \int_{0}^{w_{y}} \frac{X_{m}(x) X_{n}(x) Y_{p}(y) Y_{q}(y)}{N x_{n} N y_{q}} Z_{r}(1) \tilde{\bar{Z}}_{n q}(1) \mathrm{d} y \mathrm{~d} x$

In light of the orthogonality properties for the eigenfunctions $X_{m}(x)$ and $Y_{p}(y)$ given in Table 1 , the summations in Eq. (A4) contain only one term, as $m=n$ and as $p=q$, respectively. Therefore, Eq. (A4) reduces to:

$\bar{h}_{m p r}(0)=\bar{f}_{m p r}=-\int_{0}^{1} \tilde{\bar{Z}}_{m p}(z) Z_{r}(z) \mathrm{d} z-\sigma \tilde{\bar{Z}}_{m p}(1) Z_{r}(1)$

or in terms of the reordering index $i$

$\bar{h}_{i}(0)=\bar{f}_{i}=-\int_{0}^{1} \tilde{\bar{Z}}_{m(i) p(i)}(z) Z_{r(i)}(z) \mathrm{d} z-\sigma \tilde{\bar{Z}}_{m(i) p(i)}(1) Z_{r(i)}(1)$

\section{NOMENCLATURE}

$B^{\prime} \quad$ Aquitard thickness

$B_{1} \quad$ Thickness of streambed 1

$B_{2} \quad$ Thickness of streambed 2

$\bar{f}_{i} \quad$ Initial transformed condition

$h \quad$ Dimensionless hydraulic head

$h_{f} \quad$ Filter potential

$h_{H} \quad$ Homogeneous filtered potential
Transformed potential

$h^{*} \quad$ Hydraulic head

$H \quad$ Aquifer height

$k_{v} \quad$ Dimensionless vertical hydraulic conductivity

$k^{\prime} \quad$ Dimensionless aquitard conductivity

$k_{1}$

$k_{2}$

Dimensionless hydraulic conductivity of streambed 1 Dimensionless hydraulic conductivity of streambed 2 Aquifer horizontal hydraulic conductivity

Aquifer vertical hydraulic conductivity

Aquitard conductivity

Hydraulic conductivity of streambed 1

Hydraulic conductivity of streambed 2

Norm

Norm for the filter solution

$N_{i^{*}}$

$N x_{m}$

$N y_{p}$

$\mathrm{Nz}$

$Q$

$S$

$S_{s}$

$S_{y}$

$t$

$t^{*}$

$T$

$w_{x}$

$w_{y}$

$W_{x}$

$W_{y}$

$x \quad$ Dimensionless longitudinal coordinate

$x^{*} \quad$ Longitudinal coordinate

$x_{0}{ }^{*} \quad$ Location of the vertical well in the $\mathrm{x}$-direction

$X_{m}$

$y$

$y_{0}$

Eigenfunctions in the $\mathrm{x}$-direction defined in Table 1

Dimensionless transversal coordinate

Dimensionless location of the vertical well in the y-direction

$y^{*} \quad$ Transversal coordinate

$y_{0}{ }^{*} \quad$ Location of the vertical well in the y-direction

$Y_{p}$

Eigenfunctions in the y-direction defined in Table 1

Dimensionless vertical coordinate

Vertical coordinate

$z^{*} \quad$ Vertical coordinate

$Z_{r} \quad$ Eigenfunctions in the z-direction defined in Table 1

$\tilde{\bar{Z}}_{*} \quad$ Transformed potential for the filter solution

\section{Greek symbols}

$\alpha_{m} \quad$ Eigenvalues in the $\mathrm{x}$-direction defined in Table 1

$\beta_{p} \quad$ Eigenvalues in the $\mathrm{y}$-direction defined in Table 1

$\gamma_{r} \quad$ Eigenvalues in the $\mathrm{z}$-direction defined in Table 1

$\mu_{i} \quad$ Eigenvalues defined by Eqs. (6)

$\sigma \quad$ Ratio of aquifer specific yield to specific storage

$\phi_{i^{*}} \quad$ Eigenfunctions for the filter problem

$\psi_{i} \quad$ Eigenfunctions defined by Eqs. (6)

\section{Subscripts and superscripts}

$i, i^{*}, j \quad$ Original orders from eigenvalue problems

$k, l, m, p, r$ Derived orders from eigenvalue problems

$\ldots, \simeq \quad$ Integral transformed quantities

Received 18 October 2020 Accepted 1 July 2021 